\title{
Robot-assisted movement training for the stroke-impaired arm: Does it matter what the robot does?
}

Leonard E. Kahn, PhD; ${ }^{1-2}$ Peter S. Lum, PhD; ${ }^{3-4}$ W. Zev Rymer, MD, PhD; ${ }^{1-2}$ David J. Reinkensmeyer, PhD ${ }^{5 *}$

${ }^{1}$ Sensory Motor Performance Program, Rehabilitation Institute of Chicago, Chicago, IL; ${ }^{2}$ Departments of Biomedical Engineering and Physical Medicine and Rehabilitation, Northwestern University, Evanston, IL; ${ }^{3}$ Hunter Holmes McGuire Department of Veterans Affairs Medical Center, Richmond, VA; ${ }^{4}$ Biomedical Engineering, The Catholic University of America, Washington, DC; ${ }^{5}$ Departments of Mechanical and Aerospace Engineering and Biomedical Engineering, University of California, Irvine, CA

\begin{abstract}
Robot-assisted movement training improves arm movement ability following acute and chronic stroke. Such training involves two interacting processes: the patient trying to move and the robot applying forces to the patient's arm. A fundamental principle of motor learning is that movement practice improves motor function; the role of applied robotic forces in improving motor function is still unclear. This article reviews our work addressing this question. Our pilot study using the Assisted Rehabilitation and Measurement (ARM) Guide, a linear robotic trainer, found that mechanically assisted reaching improved motor recovery similar to unassisted reaching practice. This finding is inconclusive because of the small sample size $(n=19)$, but suggest that future studies should carefully control the amount of voluntary movement practice delivered to justify the use of robotic forces. We are optimistic that robotic forces will ultimately show additional therapeutic benefits when coupled with movement practice. We justify this optimism here by comparing results from the ARM Guide and the Mirror Image Movement Enabler robotic trainer. This comparison suggests that requiring a patient to generate specific patterns of force before allowing movement is more effective than mechanically completing movements for the patient. We describe the engineering implementation of this "guided-force training” algorithm.
\end{abstract}

Key words: arm movement, control strategies, motor control, motor learning, movement training, reaching, rehabilitation, rehabilitation therapy, robotics, stroke.

\section{INTRODUCTION: CRITIQUE OF ROBOT- ASSISTED THERAPY}

Robotic technology could partially automate movement training following injury to the central nervous system (CNS). Rehabilitation therapists spend significant time using hands-on therapy during stroke rehabilitation. Hands-on techniques, such as active-assist exercise, are advocated in practice guidelines and standard texts [1-3]. Robotic devices, because of their programmable forceproducing ability, can replicate some features of a therapist's manual assistance, allowing patients to semiautonomously practice their movement training. However, robotic devices can also implement novel forms of mechanical manipulation impossible for therapists to emulate because of limited speed, sensing, strength, and repeatability of the therapist's neuromuscular system.

Abbreviations: ARM = Assisted Rehabilitation and Measurement (Guide), CNS = central nervous system, MIME = Mirror Image Movement Enabler, MIT $=$ Massachusetts Institute of Technology.

*Address all correspondence to David J. Reinkensmeyer, PhD, Associate Professor; Department of Mechanical and Aerospace Engineering, 4200 Engineering Gateway (EG3225), University of California, Irvine, CA 92697-3975; 949-824-5218; fax: 949-824-8585. Email: dreinken@uci.edu DOI: 10.1682/JRRD.2005.03.0056 
Novel forms of manipulation may ultimately enhance movement recovery beyond current possibilities.

Since the 1997 pioneering study of Massachusetts Institute of Technology (MIT)-Manus [4], the number of research groups developing robotic therapy devices has rapidly increased. As reviewed in this issue and elsewhere [5-9], devices have been developed for automating training for arm movement following stroke, gait and posture following stroke and spinal cord injury, and wrist and finger movement following stroke. Initial results are promising: patients who receive more therapy with a robotic device recover more movement ability [9-10]. The benefits of robot-assisted therapy are comparable with or better than that of conventional therapy [11-12].

This article, however, offers an interim, yet critical, analysis of our early experiences with robot-assisted therapy. We argue that a substantial gap exists in the rationale for widespread implementation of robot-assisted therapy in rehabilitation clinics because a key question remains unanswered: "Is the expense of an actuated device needed to achieve therapeutic benefit?” Put another way, "Could similar benefits be achieved with simpler, less expensive, nonrobotic technology that facilitates movement practice?" Nonrobotic technology includes exercise machines such as hand cycles, low-cost movement monitoring, and virtual reality systems, and passive antigravity devices such as mobile arm supports and overhead slings. Clearly, a mechanical device that measures movement for directing rehabilitation is only made more expensive and less safe by adding robotic actuators. Thus, this question of the benefits of robotic actuators is practically and economically important for rehabilitation technologists and the clinicians and patients they serve. This question is also scientifically interesting, because answering it requires understanding how sensory motor activity influences CNS recovery. The answer will refine rehabilitation therapists' actions during conventional, one-on-one therapy, as well as help determine the fate of robot-assisted therapy.

We first explain why we think that this question remains unanswered, then we review two studies from our laboratories that provide clues to its answer. We focus our discussion on movement training of the arm following stroke, although similar issues are likely relevant for gait and hand training and for other CNS disorders. More comprehensive reviews of robotic therapy have been published elsewhere [5-9].

\section{Background: Initial Results with Robot-Assisted Therapy for Arm after Stroke}

The first robotic system to receive extensive clinical testing was MIT-Manus, a 2 degree-of-freedom robot manipulator that assisted patients in tabletop arm movements. In a first report, 20 stroke patients received four to five 1-hour sessions a week for up to 9 weeks with MITManus beginning on average 3 weeks after a single stroke [13]. The device assisted planar pointing and drawing movements with an impedance controller. A control group received 1-hour a week of "sham" therapy in which they used the less-impaired limb in the robot, or the robot interacted passively with the more-affected limb.

Compared with the control group, the patients who received robotic therapy had reduced shoulder and elbow motor impairment according to the Motor Status Score. The groups still statistically differed in motor impairment at a 3-year follow-up [14]. These findings were confirmed with larger samples of 56 and 96 patients [15]. Chronic stroke subjects who performed assistive and resistive exercises with MIT-Manus also improved arm movement ability [16]. This pioneering work indicates that supplemental robotic therapy can improve recovery in acute and chronic stroke patients.

The second key study of robot-assisted therapy for the arm after stroke used the Mirror Image Movement Enabler (MIME) device [6]. The MIME is a 6-degree-offreedom, industrial robot manipulator (PUMA 560 [Unimation, Inc, Connecticut, no longer in existence]) that applies forces to the paretic limb through a customized forearm splint. The robot moves the forearm through a large range of positions and orientations in threedimensional space. A six-axis sensor measures the forces and torques between the robot and the paretic limb. Several modes of robot-assisted movement have been implemented with MIME, including passive, active-assisted, and active-constrained, as well as a bimanual mode in which MIME continuously moves the impaired limb to the mirror image position of the unimpaired limb as measured with a digitizing linkage.

The initial clinical testing of MIME compared the effectiveness of robot-assisted therapy with equally intensive conventional therapy [11]. In this study, 27 chronic stroke subjects received 24 1-hour sessions over 2 months. The robot group practiced shoulder and elbow movements assisted by MIME in all four of its modes. The control group received conventional treatment and 5 minutes of robot exposure each session. At the 
conclusion of training, the robot group had statistically larger improvements in the Fugl-Meyer score, a common clinical motor impairment scale. The robot group also had larger gains in strength and reach extent. At the 6month follow-up, the groups no longer differed in FuglMeyer score; however, the robot group improved more in the self-care and transfers sections of the Functional Independence Measure. These results suggest that robotassisted therapy can be comparable with, or perhaps more effective than, conventional rehabilitation therapy.

\section{Critique of Robot-Assisted Therapy Study Designs}

While the initial MIT-Manus and MIME studies are important because they demonstrated the feasibility and potential of robot-assisted therapy, the studies did not address which components of the robot-assisted therapy produced the observed therapeutic benefits. For example, which was most beneficial-the process of the patient trying to move or of the robot applying forces to the patient's arm-remains unclear.

In the initial MIT-Manus study, the patients who received robot-assisted therapy also received more movement practice time compared with the control group. Whether these patients would have improved as much if the robot motors were turned off during their extended practice time is uncertain.

The MIME study also did not completely control the amount of movement practice in the robot and control groups. The groups received a matched therapy duration, but how much of that time the patient actually practiced movement is unclear. The finding that robot-assisted therapy was comparable with or better than conventional rehabilitation therapy is encouraging. However, this finding could also be an indictment of conventional therapy rather than proof of benefit of robotics; conventional therapy may not have maximized movement practice.

Two recent studies using MIT-Manus with chronic stroke subjects have begun to address the relative roles of robotic forces and movement practice. One study of 46 subjects found no significant differences in movement improvements for groups that received robotic assistive or resistive forces [17]. A possible explanation is that the form of robotic forces (assistive or resistive) did not matter as much as the extended movement practice, i.e., as much as the subjects trying to move. Another study with 30 subjects examined performance-based progressive therapy, an adaptive robot control strategy in which the robot intervened less if the patient was more capable
[9,18]. Impairment scores improved more with this strategy than in the previous MIT-Manus studies, especially for moderately impaired subjects for whom the robot presumably intervened less. Again, one interpretation is that the key element for stimulating recovery relates to subject effort; i.e., the progressive therapy mode may have optimized the subjects' abilities to accomplish the task while maximizing their efforts to activate damaged descending pathways and thereby reorganize those pathways.

Unassisted goal-directed practice is the predominant way for children, Olympic athletes, and stroke patients to learn a motor skill. Hands-on assistance may be used in limited circumstances for a limited time, for example, for safety during dangerous tasks or to demonstrate a desired movement. A wide body of literature has promoted the idea that "repetitively trying to achieve a goal" is important for motor learning (for a comprehensive review, see Schmidt and Lee [19]). In fact, repetitive goal-directed effort is so useful that even mental rehearsal of movement can improve arm motor impairment following stroke [20-22]. How robotic forces and quantitative feedback to the user might improve motion for persons with neurological injury beyond the benefits of repetitive practice remains unclear.

\section{CONTROLLED STUDY OF ROBOT ASSISTANCE}

We developed a device called the Assisted Rehabilitation and Measurement (ARM) Guide that measures and applies assistive or resistive forces to linear reaching movements across a wide workspace [23]. The ARM Guide consists of a hand piece that is attached to a linear track and actuated by a DC servomotor. The track can be oriented at different yaw and pitch angles to allow reaching to different workspace regions. The device is statically counterbalanced so that it does not gravitationally load the arm. We used the ARM Guide to directly test the role of movement practice versus robotic forces in retraining reaching following chronic stroke.

\section{Study Design}

We used the ARM Guide to help train reaching in chronic stroke subjects $(n=10$, mean time poststroke $=$ 6.3 years) [24-25]. Subjects in the robot-trained group performed reaching movements under their own power with active assistance from the device. The active assistance algorithm required subjects to initiate movement. 
The targeted normative movements were along a straightline path (linear track of the ARM Guide) and followed the bell-shaped velocity profile typical of unimpaired reaching movements. To emphasize the importance of subjects moving under their own efforts, we incorporated a $1 \mathrm{~cm}$ dead-band in the position trajectory that allowed a subject a small margin of error along the planned path before the motor provided assistance. Outside this deadband, the motor assisted the subject in maintaining the correct trajectory with a force proportional to a weighted sum of the position and velocity errors. To further motivate subjects to exert effort through the entire movement, we encouraged them to minimize the magnitude of robot assistance, which was provided graphically after every five movements. Control subjects ( $n=9$, mean time poststroke $=8.5$ years) practiced reaching without ARM Guide assistance. The two groups were matched by impairment level according to the arm section of the Chedoke-McMaster Stroke Assessment [26]. We included subjects who scored between 2 and 5, inclusive, on this 7-point scale.

Both training groups performed equal numbers of reaching movements (50 reaches) to identical targets, participated in sessions of equal duration (45 minutes a session, three times a week for 8 weeks), and received graphical performance feedback throughout each session. However, only the robot-trained group received mechanical assistance to complete the desired movement.

We evaluated the subjects' movement ability using various outcome measures, including measures of reaching and functional task performance [24-25]. The most sensitive measures were derived from a test of the subjects' supported reaching ability. At the beginning of each of the 24 training sessions, subjects in both groups reached as far and fast as possible along the horizontal ARM Guide track and we measured their range and maximum speed of movement.

\section{Summary of ARM Guide Study Results}

Both groups significantly improved their maximum speed during supported reaching gradually across training sessions, but improvements did not significantly differ between the groups (Figure 1). Significant improvements with training, but not between training methods, were also observed for the maximum range of supported movement. Of particular interest to both clinicians and patients was that these gains translated into improved performance of functional tasks (decreased time to complete each task) on the Rancho Los Amigos Functional Test of the Hemiparetic Upper Extremity [27]. Tasks included practical activities of daily living like zipping a jacket, putting a pillow in a pillowcase, and wringing a rag. Limb stiffness and movement range of the hand as it reached in free space to a target did not change after training. The discrepant results between the measures might be explained by the fact that reaching out from the body in free space is a relatively demanding task that requires substantial shoulder strength. Many functional tasks are achievable with partial support of the arm resting on a table or with the arm closer to the body.

\section{Discussion of ARM Guide Study}

In this study, subjects who received robotic assistance exhibited improved arm movement ability similar to that of subjects who received no assistance. This result surprised us: the active-assist exercise received by the robot-

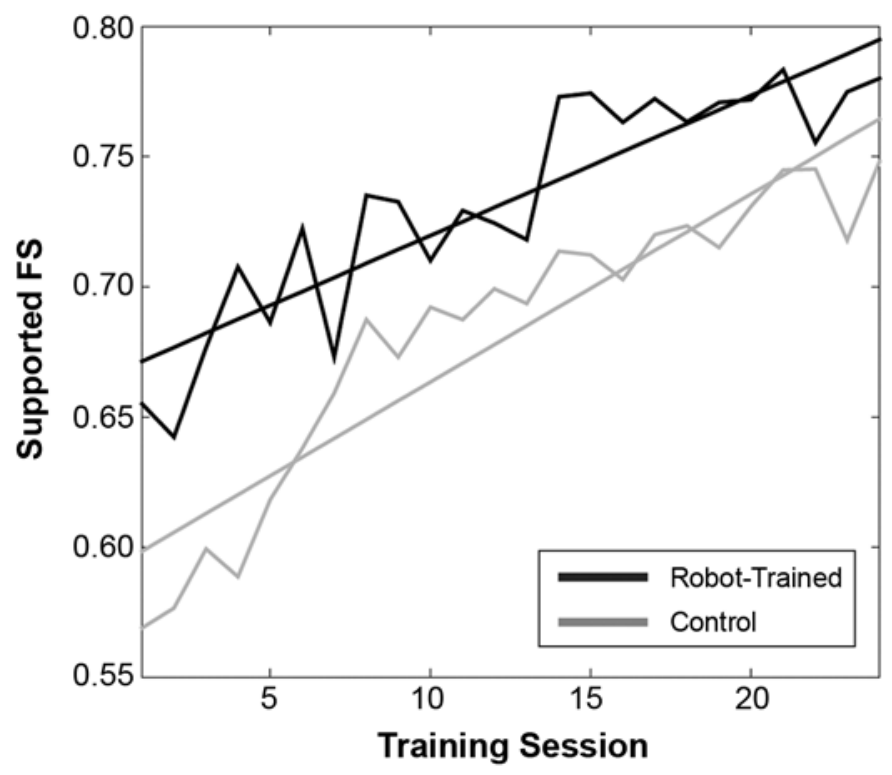

Figure 1.

Comparison of supported fraction of speed (FS) for robot-trained group and control group, which trained without robot assistance. FS is speed during fast-as-possible reaching along Assisted Rehabilitation and Measurement Guide by hemiparetic arm, divided by speed of contralateral arm, with device oriented horizontally. Ensemble averages across subjects in each group and regression lines (robottrained: $R^{2}=0.79, p<0.001$; control: $R^{2}=0.86, p<0.001$ ) shown for 24 training sessions over 8-week period. Adapted from Kahn LE, Zygman ML, Rymer WZ, Reinkensmeyer DJ. Robot-assisted reaching exercise promotes arm movement recovery in chronic hemiparetic stroke: A randomized controlled pilot study. J Neuroengineering Rehabil. 2006;3:12. 
trained group forcefully extended the arm as each subject exerted effort to reach forward. We thought such stretching would at least improve movement ability by reducing passive tone and spasticity, and perhaps, by providing novel somatosensory input that stimulated neural reorganization. The observed gains, possibly indicative of such plasticity, were anticipated to be greater than those attained from repetitive reaching without assistance.

One possible explanation for the lack of a differential benefit for active-assist exercise is that the sample size was small and a difference went undetected. Longitudinal power analysis is a powerful technique for calculating the probability of such an error, given data from many measurements over time [28]. We applied longitudinal power analysis techniques to the maximum supported velocity data from each training session (Figure 1) and found that the study had an 80 percent chance to detect a 30 percent difference in the maximum supported velocity. Thus, any difference between training techniques for this measure was likely incremental rather than dramatic. Incidentally, we believe that maximum supported velocity of ballistic reaching is a promising measure of recovery because it is simple, inexpensive, quantitative, functionally relevant, and most importantly, sensitive to movement ability across the wide range of impairment levels used in this study. This measure does not appear to have either floor effects, because even very impaired patients can slide their arms along a support, or ceiling effects, because less-impaired patients can learn to move faster.

Another possible conclusion from these results is that the "robot" in our "robot-assisted therapy" program was superfluous. However, this study tested only one possible form of robotic therapy using a specific device with a small sample of chronic stroke subjects over a limited number of repetitions. Thus, while these results confirm that movement practice is a primary stimulant for movement recovery (both groups did make movement gains) and lead us to postulate that robotically assisting completion of a movement for a chronic stroke subject does not have a strong therapeutic benefit within the number of repetitions tested, another pattern of robotic forces or a greater level of exposure to robotic forces could possibly act synergistically with the process of movement practice.

\section{COMPARISON OF RESULTS FROM MIME AND ARM GUIDE: IT DOES MATTER WHAT THE ROBOT DOES}

In the first therapeutic study of the MIME, robotassisted therapy resulted in larger gains in reach extent than conventional therapy. Reach extent was defined as the distance that a patient could reach unassisted toward a target (i.e., unsupported active range of motion of reaching). Reach extent was also an outcome measure in the ARM Guide study, allowing comparison [29]. The key finding was that subjects who participated in nonrobotic therapy (unassisted reaching in the ARM Guide study or conventional rehabilitation therapy in the MIME study) and subjects who received active assistance from the ARM Guide did not improve their reach extent. Only subjects who received movement training with the MIME improved their reach extent (Figure 2).

Three possible causes for this difference in reach extent are therapy intensity, kinematics of practiced movements, and therapy modes. The MIME subjects experienced 24, 50-minute sessions over 8 weeks, compared with 24, 45-minute sessions over 8 weeks for ARM Guide subjects. Subjects performed movements at

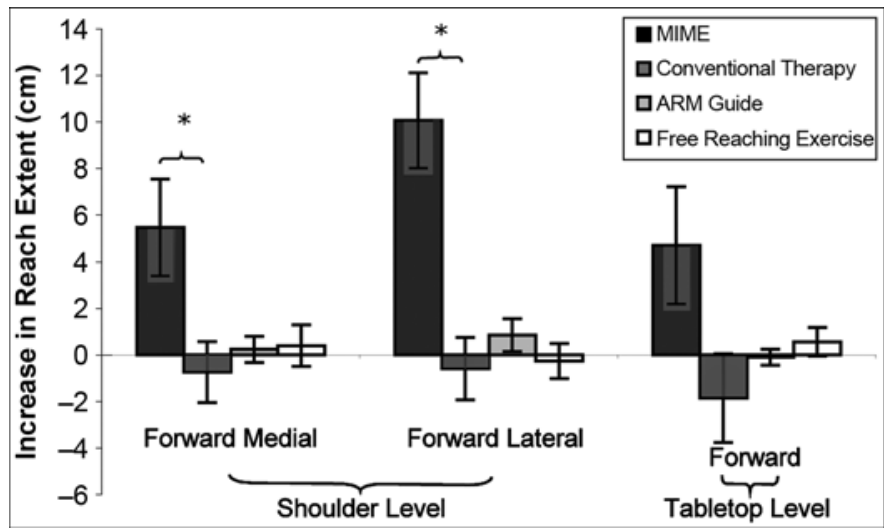

Figure 2.

Comparison of improvement in reaching extent for treatment groups from Mirror Image Movement Enabler (MIME) and Assisted Rehabilitation and Measurement (ARM) Guide studies. Only MIME group improved reach extent. Error bars indicate standard deviation. ${ }^{*}$ Significant difference between groups, $p<0.05$. Adapted from Kahn LE, Reinkensmeyer DJ. Selection of robotic therapy algorithms for the upper extremity in chronic stroke: Insights from MIME and ARM Guide results. Proceedings of the International Conference on Rehabilitation Robotics; 2003 Apr 23-25; Daejeon, Korea. Daejeon (Korea): Human-Friendly Welfare Robot System Engineering Research Center; 2003; p. 208-10. 
approximately the same frequency during these sessions. Consequently, we believe that this dissimilarity in therapy intensity was too small to cause the highly significant improvements with the MIME but not the ARM Guide.

Another possible explanation is that the movements practiced with the MIME differed from other protocols. The MIME accommodates fully naturalistic arm postures during reaching because of its 6 degrees of freedom, while the ARM Guide constrains arm movement to a linear path. However, we assessed reaching movements to obtain the reach extent outcome measure of interest here. The devices showed a small difference in kinematics for reaching movements, since reaching movements follow approximately straight-line paths. Thus, different arm postures during training seem unlikely to account for the different reach extent outcomes.

Lastly, two modes of active therapy were included in the MIME study but not the ARM Guide study. First, the training of bimanual mirror movements may have provided a unique stimulus for recovery of bilateral or ipsilesional neuromotor pathways. Recent bimanual therapy studies have shown some transfer to unimanual tasks [30-32]. However, preliminary data from a controlled study with the MIME comparing bimanual to unimanual therapy suggest that the end benefits achieved by each mode are similar [33]. Second, in active-constrained mode, a force sensor measured the direction of force generated by the subject's hand at the interface between the hand and the robot. If the force vector had a component in the desired direction (i.e., toward the target), then the robot moved in that direction with a velocity proportional to force. If the force was misdirected, however, the robot stopped moving toward the target and a programmed impedance allowed the robot to deflect slightly in the direction of the force providing visual feedback of the misdirection. This training mode forced subjects to not only activate muscles to move the limb but also to try to activate muscle groups in appropriate combinations, depending on the desired target and the limb configuration.

\section{GUIDED-FORCE TRAINING HYPOTHESIS}

The active-constrained mode used by MIME is one example of "guided-force training" that requires a patient to generate specific patterns of force to move. We hypothesize that guided-force training accounts for the difference between the two studies because it helped sub- jects efficiently relearn the sensory motor transformations required for reaching. Essentially, the robot halted the subjects' movements when it sensed grossly incorrect muscle activation patterns, forcing subjects to attempt to generate a more normative pattern at each troublesome workspace position.

Generating the correct pattern required lifting the arm against gravity, which likely strengthened the arm. Moreover, the nature of the task required significant attention and effort, both of which predict the amount of motor learning in a task [19,34-35]. If the subjects correctly sequenced the learned patterns, they were rewarded with a smooth, uninterrupted movement toward the target. The active-assist mode used exclusively with the ARM Guide helped the subjects move through their full passive range of motion but did not allow the subjects to systematically decompose and revise incorrect muscle activations. This mode also did not penalize subjects for allowing the device to support the arm as they moved.

Individuals with a stroke often display anisotropic or nonuniform patterns of weakness: when asked to generate isometric force in a specific direction, they are surprisingly strong in some directions but dramatically weak in others [36]. Anisotropic weakness may arise from constrained muscle-activation pathways [36-37] or muscle strength imbalances [38]. Either way, requiring subjects to exercise in their weakest directions makes sense for enhancing the strength required for function [36]. Guided-force training provides directionally targeted strength training across the arm's workspace. This technique would be extremely challenging for a therapist to implement. Implementing guided-force training nonrobotically may be possible with mechanical guides and ratcheting mechanisms, but robotic devices provide a convenient and flexible way to initially assess its value.

\section{IMPLEMENTING GUIDED-FORCE TRAINING}

Given the inconclusive result of our first ARM Guide study regarding therapeutic benefits of robotic forces, we have begun a second ARM Guide study to test whether guided-force training differentially benefits chronic stroke subjects, compared with unassisted reaching exercise [39]. This section describes the engineering design and initial testing of the guided-force training algorithm. Therapeutic testing of the algorithm is still under way and will not be reported. 


\section{Guided-Force Training Algorithm}

Guided-force training promotes conscious shaping of endpoint forces in the hemiparetic arm. We make the subject aware of the forces generated against the ARM Guide by measuring those forces with a six-axis force transducer attached to the hand piece. During a single trial, the user attempts to reach forward at a comfortable speed to the end of his or her range of motion. A graphical interface on a computer monitor represents the current position of the ARM Guide and a target position at the end of the passive range of motion. Throughout the movement, the force transducer is monitored for off-axis forces (i.e., forces perpendicular to the desired movement direction) above a $10 \mathrm{~N}$ threshold, a value selected based on data from unimpaired subjects. Once such a force is detected, the motor locks the position of the hand piece and the user receives real-time graphical feedback of the error. The user is instructed to relax, then begin a new reaching movement from the current position taking into account the forceerror feedback. When the force transducer detects appropriate forces toward the target, the graphical cue is removed and the motor unlocks the hand piece, which allows the user to progress toward the target. Essentially, the control algorithm breaks the reach into a series of discrete movements bounded by error events. Our goal is to force users to learn how to generate the correct muscle activations at troublesome workspace positions.

One issue we encountered in designing the guidedforce training program was that the target population of chronic stroke patients exhibits a wide range of arm impairment levels. Some subjects can move through a large range of motion at a high velocity, while others have severe range and velocity limitations with stronger coupling of motions between the elbow and shoulder (e.g., elbow flexion is uncontrollably elicited with shoulder abduction). To account for varying movement abilities, we designed the training such that the threshold at which off-axis forces lock the hand piece can be raised so that the reaching task is achievable but still provides challenge and feedback to the subject. Furthermore, adapting the level of assistance provided during guidedforce training on a patient-specific basis is desirable because each patient could practice movements with maximal achievable reaching range and speed and minimal robotic assistance. In our experience, making the task "difficult but doable" is important for maximizing subject motivation.
We adapted the training to each subject's ability by adding a velocity-dependent assistive force from the motor. The difference between the maximum velocity of the previous reach and a desired velocity (quantified as the maximum speed of a self-paced "comfortable" movement with the ipsilesional arm unattached to the device) determines a coefficient $b$ that specifies the assistive (or resistive) force for the next movement:

$$
b_{i}=b_{i-1}+c\left\{\max \left[v_{\mathrm{ipsi}}(t)\right]\right\}-\max \left[v_{i-1}(t)\right],
$$

where $b_{i}$ is the velocity assistance coefficient for trial $i$, $c$ is an adaptation constant determining increment size, $v_{i-1}(t)$ is the velocity profile of the previous trial, and $v_{\text {ipsi }}(t)$ is the velocity profile of the ipsilesional arm. During trial $i$, the coefficient is multiplied by the instantaneous velocity to determine motor output:

$$
m_{i}(t)=b_{i} v_{i}(t)
$$

Thus, the motor does not assist movement unless the subject initiates movement. Even in subjects who have very little ability to move their paretic arm, the assistance provided destabilizes the subjects' movement, increasing the range and speed. As the motor assistance increases the subject's movement velocity, the algorithm lowers the velocity assistance coefficient $b_{i}$ from trial to trial until the actual and desired peak movement speeds are equal. If the subject is able to move faster than the desired velocity, the algorithm decreases the velocity assistance and eventually resists movement if $b_{i}$ becomes negative. The algorithm is compatible with the guided-force training because the assistance is velocity-dependent. Thus, if the subject's movement is stopped because of an errant off-axis force, the assistance is terminated. When the subject generates forces in the correct direction, the assistance turns on smoothly.

\section{Example Use of Guided-Force Training Algorithm}

To illustrate the feasibility of the guided-force training and adaptive assistance, we had two chronic stroke subjects and an unimpaired subject practice 15 reaching movements to a single target in a single session. To examine repeatability of the algorithm, we asked one of the chronic stroke subjects to practice 15 movements to the same target for 4 days. As shown in Figure 3, the coefficient of assistance converged to a consistent value for all three subjects. The coefficient for severely 

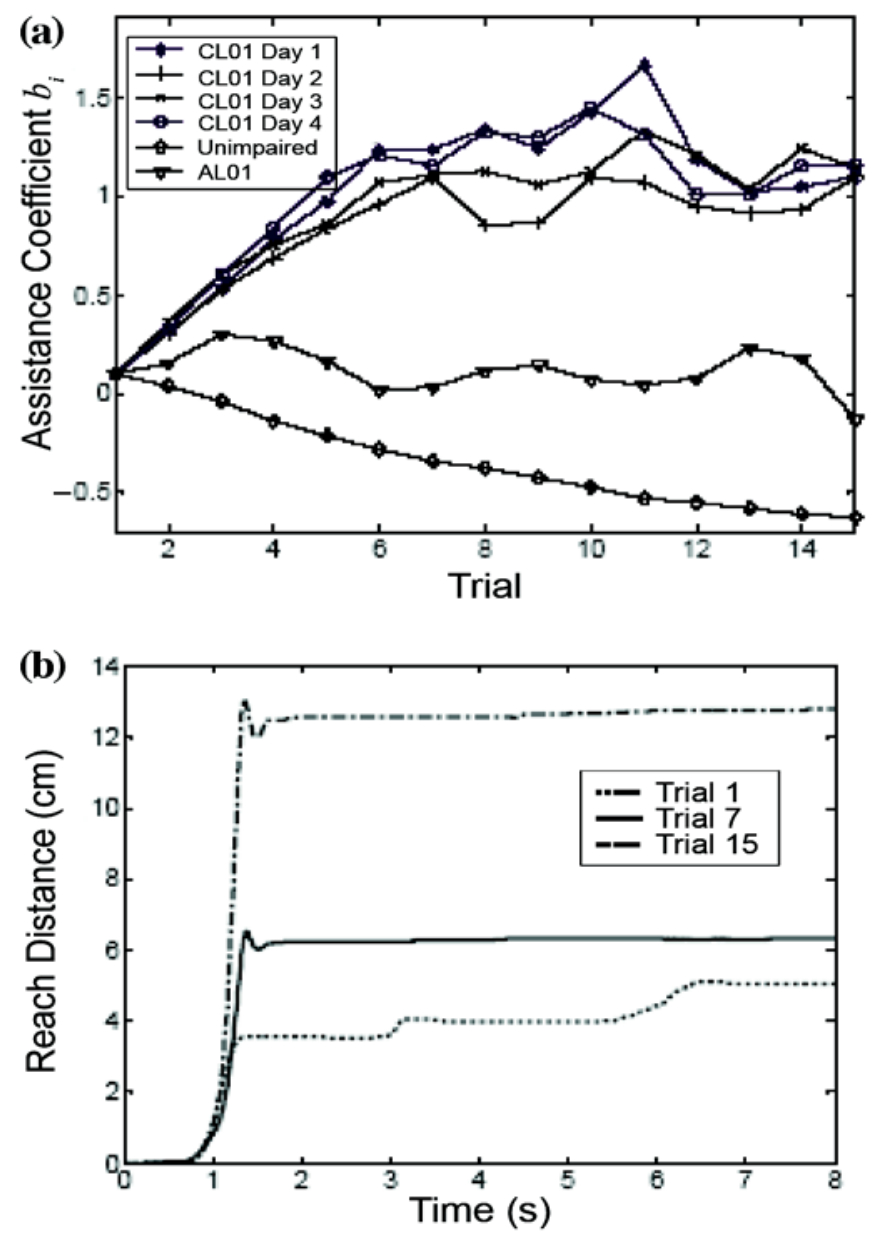

Figure 3.

Adaptive guided-force training algorithm. (a) Convergence of assistance over multiple reaching trials along Assisted Rehabilitation and Measurement Guide. Values for stroke subject CL01 consistently converged around single value ( 1.1) over multiple sessions on different days. Values for single session also shown for stroke subject AL01 and unimpaired subject. (b) Increasing assistance from device allowed subject to reach farther. Trajectories shown for stroke subject CL01's reaching trials 1, 7, and 15 on Day 4. Reprinted with partial alteration by permission from Kahn LE, Rymer WZ, Reinkensmeyer DJ. Adaptive assistance for guided force training in chronic stroke. Proceedings of the 26th Annual IEEE Engineering in Medicine and Biology Society Meeting; 2004 Sep 1-5; San Francisco, CA. Piscataway, NJ: IEEE press; 2004. p. 2722-25. (C [2004] IEEE.)

impaired stroke subject CL01 repeatedly approached similar values on multiple visits to the laboratory when this subject was reaching to the same target. The coefficient converged on a lower value for subject AL01 who displayed greater functional ability on a clinical scale (3 out of 7 on the Chedoke-McMaster Stroke Assessment) than subject CL01 (2 out of 7). The coefficient became negative for an uninjured subject who was able to move at a velocity greater than the desired velocity. Figure 3 also shows the desired effect of allowing a severely impaired subject to gradually reach faster and farther by automatically increasing assistance based on the sensed error and the algorithm given by Equations (1) and (2).

\section{Discussion of Guided-Force Training Algorithm}

These results demonstrate the feasibility of a guidedforce training paradigm with adaptive assistance. This type of training requires the subject to generate normative force patterns to move, but it also adapts to the subject's ability based on velocity measurements. Thus, even severely impaired subjects achieve a sense of satisfaction in completing the desired reaching task with short-term practice (tens of reaches), although they still must work very hard at trying to move. The algorithm is different from the original MIME active-constrained mode because it requires a more focused force generation (within a forward-facing cylinder in force space rather than just some force component toward the target), which adds the potential advantage of requiring greater attention from the subject. Guided-force training also departs from the MIME because it adapts the assistance (or resistance) based on the subject's performance. We are currently testing this training algorithm in a study that compares its benefits to a repetition-matched unassisted reaching exercise and a time-matched conventional therapy. The current guided-force training paradigm with the ARM Guide combines visual, haptic, and proprioceptive cues. Future studies will examine the roles of these sensory cues as well as possible synergistic effects. Recent studies suggest that manipulating tactile/haptic [40-44] and visual cues [45-46] can enhance learning.

\section{CONCLUSIONS}

Distinguishing the benefits of the two interacting processes highlighted here-movement practice and application of robotic forces-is critical for determining the future of robot-assisted therapy. If movement practice is the dominant stimulus for movement recovery, then robotic actuators may turn out to be technological ornamentation. The question remains whether complex, and potentially expensive, devices are essential for maximizing the learning and recovery capabilities of the injured CNS, or if less complex — and likely less expensivemachines without actuation [47-48] that facilitate optimal 
forms and amounts of practice will be the most viable solution. In the interim, robotic devices are useful tools for rigorously addressing the role of external forces on neurorecovery. These devices also allow strictly controlled comparison of training effects across intervention methods, severity levels, lesion locations, time postinjury, sex, and virtually any other patient parameter. They will provide a useful tool for understanding the specific mechanisms of neuroplasticity that support motor learning after stroke, although this area has been largely unaddressed in the context of robotic therapy.

We can clarify the role of robotic forces by including control groups that do not use robotic forces. For example, the control group that received a matched amount of reaching practice but no robotic forces in the ARM Guide study provided insight into whether the specific form of robotic forces was necessary for motor gains. Our conclusion is that robotically finishing a movement for a chronic stroke subject did not add value beyond the concurrent movement practice. Thus, our advice for clinicians considering bearing the additional costs of robotic technology is to wait for further controlled testing, particularly if they will use that technology only to finish patient-initiated movements.

Nonetheless, based on our comparison of the MIME and ARM Guide results, we are optimistic that robotic forces will be found useful. Guided-force training techniques hold promise for improving arm movement ability after stroke because they directly address anisotropic weakness, a key impairment after stroke. Guided-force training uses robotic forces to focus and intensify patient effort and attention. We expect the principle of using robotic forces to optimize subject effort to be important in the future development of robot-assisted therapy.

\section{ACKNOWLEDGMENTS}

This material was based on work supported in part by the U.S. Department of Education National Institute on Disability and Rehabilitation Research (NIDRR) Field-Initiated grant H133G80052; NIDRR Rehabilitation Engineering Research Center on Rehabilitation Robotics and Telemanipulation grant H133E020724; National Institutes of Health, National Institute of Child Health and Human Development, Institutional National Research Service Award (Rymer); and Department of Veterans Affairs merit review grant B2056RA.
The authors have declared that no competing interests exist.

\section{REFERENCES}

1. American Physical Therapy Association. Guide to physical therapist practice. 2nd ed. Alexandria (VA): American Physical Therapy Association; 2001.

2. Bobath B. Adult hemiplegia: Evaluation and treatment. 2nd ed. London (England): William Heinemann Medical Books, Ltd; 1978.

3. Ryerson SD. Hemiplegia. In: Umphred DA, editor. Neurological rehabilitation. 3rd ed. St. Louis (MO): The C. V. Mosby Company; 1995. p. 681-721.

4. Aisen ML, Krebs HI, Hogan N, McDowell F, Volpe BT. The effect of robot-assisted therapy and rehabilitative training on motor recovery following stroke. Arch Neurol. 1997;54(4):443-46. [PMID: 9109746$]$

5. Hesse S, Schmidt H, Werner C, Bardeleben A. Upper and lower extremity robotic devices for rehabilitation and for studying motor control. Curr Opin Neurol. 2003;16(6): 705-10. [PMID: 14624080]

6. Riener R, Nef T, Colombo G. Robot-aided neurorehabilitation of the upper extremities. Med Biol Eng Comput. 2005; 43(1):2-10. [PMID: 15742713]

7. Volpe BT, Krebs HI, Hogan N. Robot-aided sensorimotor training in stroke rehabilitation. Adv Neurol. 2003;92:429-33. [PMID: 12760210]

8. Reinkensmeyer DJ, Emken JL, Cramer SC. Robotics, motor learning, and neurological recovery. Ann Rev Biomed Eng. 2004;6:497-525. [PMID: 15255778]

9. Hogan N, Krebs HI. Interactive robots for neuro-rehabilitation. Restor Neurol Neurosci. 2004;22(3-5):349-58. [PMID: 15502275]

10. Hesse S, Schulte-Tigges G, Konrad M, Bardeleben A, Werner C. Robot-assisted arm trainer for the passive and active practice of bilateral forearm and wrist movements in hemiparetic subjects. Arch Phys Med Rehabil. 2003; 84(6):915-20. [PMID: 12808550]

11. Lum PS, Burgar CG, Shor PC, Majmundar M, Van der Loos M. Robot-assisted movement training compared with conventional therapy techniques for the rehabilitation of upper-limb motor function after stroke. Arch Phys Med Rehabil. 2002;83(7):952-59. [PMID: 12098155]

12. Werner C, Von Frankenberg S, Treig T, Konrad M, Hesse S. Treadmill training with partial body weight support and an electromechanical gait trainer for restoration of gait in subacute stroke patients: A randomized crossover study. Stroke. 2002;33(12):2895-2901. [PMID: 12468788] 
13. Krebs HI, Hogan N, Aisen ML, Volpe BT. Robot-aided neurorehabilitation. IEEE Trans Rehabil Eng. 1998;6(1): 75-87. [PMID: 9535526]

14. Volpe BT, Krebs HI, Hogan N, Edelstein L, Diels CM, Aisen ML. Robot training enhanced motor outcome in patients with stroke maintained over 3 years. Neurology. 1999;53(8):1874-76. [PMID: 10563646]

15. Volpe BT, Krebs HI, Hogan N, Edelstein L, Diels C, Aisen ML. A novel approach to stroke rehabilitation: Robot-aided sensorimotor stimulation. Neurology. 2000;54(10):1938-44. [PMID: 10822433]

16. Fasoli SE, Krebs HI, Stein J, Frontera WR, Hogan N. Effects of robotic therapy on motor impairment and recovery in chronic stroke. Arch Phys Med Rehab. 2003; 84(4):477-82. [PMID: 12690583]

17. Stein J, Krebs HI, Frontera WR, Fasoli SE, Hughes R, Hogan N. Comparison of two techniques of robot-aided upper limb exercise training after stroke. Am J Phys Med Rehabil. 2004;83(9):720-28. [PMID: 15314537]

18. Ferraro M, Palazzolo JJ, Krol J, Krebs HI, Hogan N, Volpe BT. Robot-aided sensorimotor arm training improves outcome in patients with chronic stroke. Neurology. 2003; 61(11):1604-7. [PMID: 14663051]

19. Schmidt RA, Lee TD. Motor control and learning: A behavioral emphasis. 4th ed. Champaign (IL): Human Kinetics; 2005.

20. Page SJ, Levine P, Sisto S, Johnston MV. A randomized efficacy and feasibility study of imagery in acute stroke. Clin Rehabil. 2001;15(3):233-40. [PMID: 11386392]

21. Stevens JA, Stoykov ME. Using motor imagery in the rehabilitation of hemiparesis. Arch Phys Med Rehabil. 2003; 84(7):1090-92. [PMID: 12881842]

22. Dijkerman HC, Ietswaart M, Johnston M, MacWalter RS. Does motor imagery training improve hand function in chronic stroke patients? A pilot study. Clin Rehabil. 2004;18(5):538-49. [PMID: 15293488]

23. Reinkensmeyer DJ, Kahn LE, Averbuch M, McKennaCole A, Schmit BD, Rymer WZ. Understanding and treating arm movement impairment after chronic brain injury: Progress with the ARM Guide. J Rehabil Res Dev. 2000;37(6):653-62. [PMID: 11321001]

24. Kahn LE, Averbuch M, Rymer WZ, Reinkensmeyer DJ. Comparison of robot-assisted reaching to free reaching in promoting recovery from chronic stroke. In: Mokhtari M, editor. Integration of assistive technology in the information age. Amsterdam (the Netherlands): IOS Press; 2001. p. 39-44.

25. Kahn LE, Zygman ML, Rymer WZ, Reinkensmeyer DJ. Robot-assisted reaching exercise promotes arm recovery in chronic hemiparetic stroke: A randomized controlled pilot study. J Neuroengineering Rehabil. 2006;3:12.

26. Gowland C, Stratford P, Ward M, Moreland J, Torresin W, Van Hullenaar S, Sanford J, Barreca S, Vanspall B, Plews
N. Measuring physical impairment and disability with the Chedoke-McMaster Stroke Assessment. Stroke. 1993; 24(1):58-63. [PMID: 8418551]

27. Wilson DJ, Baker LL, Craddock JA. Functional test for the hemiparetic upper extremity. Am J Occup Ther. 1984; 38(3):159-64. [PMID: 6711667]

28. Diggle P, Heagerty P, Liang KY, Zeger SL. Analysis of longitudinal data. 2nd ed. Oxford (England): Oxford University Press; 2002.

29. Kahn LE, Lum PS, Reinkensmeyer DJ. Selection of robotic therapy algorithms for the upper extremity in chronic stroke: Insights from MIME and ARM Guide results. Proceedings of the 8th International Conference on Rehabilitation Robotics; 2003 Apr 23-25; Daejeon Korea. Daejeon (Korea): Human-Friendly Welfare Robot System Engineering Research Center; 2003. p. 208-10.

30. Luft AR, McCombe-Waller S, Whitall J, Forrester LW, Macko R, Sorkin JD, Schulz JB, Goldberg AP, Hanley DF. Repetitive bilateral arm training and motor cortex activation in chronic stroke: A randomized controlled trial. JAMA. 2004;292(15):1853-61. [PMID: 15492483] Erratum in: JAMA. 2004;292(20):2470.

31. Cauraugh JH, Kim S. Two coupled motor recovery protocols are better than one: Electromyogram-triggered neuromuscular stimulation and bilateral movements. Stroke. 2002;33(6):1589-94. [PMID: 12052996]

32. Whitall J, McCombe Waller S, Silver KH, Macko RF. Repetitive bilateral arm training with rhythmic auditory cueing improves motor function in chronic hemiparetic stroke. Stroke. 2000;31(10):2390-95. [PMID: 11022069]

33. Lum PS, Burgar CG, Van der Loos M, Shor PC, Majmundar M, Yap R. The MIME robotic system for upper-limb neuro-rehabilitation: Results from a clinical trial in subacute stroke. Proceedings of the IEEE 9th International Conference on Rehabilitation Robotics; 2005 Jun 28-Jul 1; Chicago, IL. Piscataway (NJ): IEEE Press; 2005. p. 511-14.

34. Robertson IH, Ridgeway V, Greenfield E, Parr A. Motor recovery after stroke depends on intact sustained attention: A 2-year follow-up study. Neuropsychology. 1997;11(2): 290-95. [PMID: 9110335]

35. Pohl PS, Filion DL, Kim SH. Effects of practice and unpredictable distractors on planning and executing aiming after stroke. Neurorehabil Neural Repair. 2003;17(2):93-100. [PMID: 12814054]

36. Dewald JP, Beer RF. Abnormal joint torque patterns in the paretic upper limb of subjects with hemiparesis. Muscle Nerve. 2001;24(2):273-83. [PMID: 11180211]

37. Dewald JP, Pope PS, Given JD, Buchanan TS, Rymer WZ. Abnormal muscle coactivation patterns during isometric torque generation at the elbow and shoulder in hemiparetic subjects. Brain. 1995;118(Pt 2):495-510. [PMID: 7735890] 
38. Lum PS, Burgar CG, Shor PC. Evidence for strength imbalances as a significant contributor to abnormal synergies in hemiparetic subjects. Muscle Nerve. 2003;27(2): 211-21. [PMID: 12548529]

39. Kahn LE, Rymer WZ, Reinkensmeyer DJ. Adaptive assistance for guided force training in chronic stroke. Proceedings of the 26th Annual IEEE Engineering in Medicine and Biology Society Meeting, Vol 4; 2004 Sep 1-5; San Francisco, CA. Piscataway (NJ): IEEE Press; 2004. p. 2722-25.

40. Liu J, Cramer SC, Reinkensmeyer DJ. Learning to perform a new movement with robotic assistance: Comparison of haptic guidance and visual demonstration. J Neuroengineering Rehabil. 2006;3(1):20.

41. Patton JL, Mussa-Ivaldi FA, Rymer WZ. Altering movement patterns in healthy and brain-injured subjects via custom designed robotic forces. Proceedings of the 23rd Annual IEEE Engineering in Medicine and Biology Society Meeting. Vol. 2; 2001 Oct 25-29; Istanbul, Turkey. Piscataway (NJ): IEEE Press; 2001. p. 1356-59.

42. Feygin D, Keehner M, Tendick F. Haptic guidance: Experimental evaluation of a haptic training method for a perceptual motor skill. Proceedings of the 10th International Symposium on Haptic Interfaces for Virtual Environment and Teleoperator Systems; 2002 Mar 24-25; Orlando, FL. Piscataway (NJ): IEEE Press; 2002. p. 40-47.

43. Henriques DY, Flanders M, Soechting JF. Haptic synthesis of shapes and sequences. J Neurophysiol. 2004;91(4): 1808-21. [PMID: 14645382]
44. Emken JL, Reinkensmeyer DJ. Robot-enhanced motor learning: Accelerating internal model formation during locomotion by transient dynamic amplification. IEEE Trans Neural Syst Rehabil Eng. 2005;13(1):33-39. [PMID: 15813404]

45. Wei Y, Bajaj P, Scheidt R, Patton JL. Visual error augmentation for enhancing motor learning and rehabilitative relearning. Proceedings of the IEEE 9th International Conference on Rehabilitation Robotics; 2005 Jun 28-Jul 1; Chicago, IL. Piscataway (NJ): IEEE Press; 2005. p. 505-10.

46. Brewer BR, Fagan M, Klatzky RL, Matsuoka Y. Perceptual limits for a robotic rehabilitation environment using visual feedback distortion. IEEE Trans Neural Syst Rehabil Eng. 2005;13(1):1-11. [PMID: 15813400]

47. Sanchez R, Reinkensmeyer DJ, Shah P, Liu J, Rao S, Smith R, Cramer S, Rahman T, Bobrow J. Monitoring functional arm movement for home-based therapy after stroke. Proceedings of the 26th Annual IEEE Engineering in Medicine and Biology Society Meeting, Vol. 7; 2004 Sep 1-5; San Francisco, CA. Piscataway (NJ): IEEE Press; 2004. p. 4787-90.

48. Lum PS, Taub E, Schwandt D, Postman M, Hardin P, Uswatte G. Automated Constraint-Induced Therapy Extension (AutoCITE) for movement deficits after stroke. J Rehabil Res Dev. 2004;41(3A):249-58. [PMID: 15543442]

Submitted for publication March 2, 2005. Accepted in revised form August 16, 2005. 